\title{
Differentiation of human pre-adipocytes by recombinant adiponectin
}

\author{
Maria do Carmo Avides*, Lucília Domingues, António Vicente, José Teixeira \\ Department of Biological Engineering, IBB, Institute for Biotechnology and Bioengineering, University of Minho, \\ Campus of Gualtar, 4710-057 Braga, Minho, Portugal
}

Received 15 November 2007, and in revised form 3 January 2008

Available online 26 January 2008

\begin{abstract}
Obesity has become a global health problem and it is linked to a higher risk of diseases and metabolic disorders such as diabetes, cardiovascular disease, and cancer. The adipose tissue plays an important role in monitoring and controlling whole-body metabolism by secreting a variety of bioactive molecules such as adiponectin. Deficiencies of this hormone can cause type II diabetes and cardiovascular disease both in mice and in humans. Therefore, adiponectin is an attractive molecule to use in human therapy, particularly in a recombinant form. The source of recombinant adiponectin could be the expression of full-length adiponectin either in Escherichia coli, or in baculovirus. In this work we express and purify human adiponectin in both systems. The adiponectin produced by baculovirus was found to be 10 times more active as far as oligomerization and human pre-adipocyte differentiation are concerned, when compared with adiponectin produced by E. coli. We presume that adiponectin expressed in baculovirus has post-translation modifications not made by bacteria which may be responsible for these differences in activity. This renders adiponectin produced by baculovirus a better candidate for the treatment of type II diabetes and cardiovascular disease.
\end{abstract}

(c) 2008 Elsevier Inc. All rights reserved.

Keywords: Active adiponectin; Baculovirus; Adipocytes; Differentiation; Hormone

Obesity, the most common nutritional disease in industrial countries, is associated with increased cardiovascular mortality and morbidity $[1-4,15]$. Visceral fat accumulation is correlated to the development of cardiovascular disease and obesity-related disorders such as type II diabetes mellitus, hyperlipidemia, and hypertension [5]. The coexistence of these diseases has been termed the metabolic syndrome and insulin resistance is a key feature of this syndrome [4]. Adipose tissue plays major functions in energy homeostasis, lipid metabolism, and insulin actions by regulating the secretion of diverse factors such as leptin, adiponectin, tumor necrosis factor- $\alpha(\mathrm{TNF}-\alpha)^{1}$, plasminogen activator inhibitor-1, and various cytokines [5]. Adipocyte differentiation

\footnotetext{
* Corresponding author. Fax: +351 253678986.

E-mail address: carmoavides@deb.uminho.pt (M. do Carmo Avides).

${ }^{1}$ Abbreviations used: TNF- $\alpha$, tumor necrosis factor- $\alpha$; C/EBP, CCAAT/ enhancer-binding proteins; PPAR- $\gamma$, peroxisome proliferators-activated receptor- $\gamma$; PI3K, phosphoinositide-3-kinase; LMW, low molecular weight; MMW, middle molecular weight; HMW, high molecular weight.
}

is critical for metabolic homeostasis and nutrient signaling. Treatment of pre-adipocytes with insulin, glucocorticoids, and cAMP inducers, such as adiponectin, stimulates a transcriptional cascade resulting in a gene expression profile specific for adipocyte function [6]. Several critical transcription factors and cross-talk between those factors have been well defined, including the CCAAT/enhancer-binding proteins $(\mathrm{C} / \mathrm{EBP})$ and peroxisome proliferators-activated receptor- $\gamma$ (PPAR- $\gamma$ ). The phosphoinositide-3-kinase (PI3K)/Akt pathway has also been shown to be critically involved in adipocyte differentiation. The activity of PI3K and Akt kinases is increased during the differentiation process [7], and constitutive activation of Akt leads to spontaneous differentiation without hormone stimulation.

Adiponectin/Acrp30 is present in human plasma and breast milk and is critical in the control of whole-body metabolism, particularly by enhancing insulin sensitivity in muscle and liver, and by increasing fatty acid oxidation in muscle [8-12]. Adiponectin is a 244 amino acid protein composed of four distinct domains: A signal peptide at 
the N-terminus, a short variable region, a collagenous domain, and a C-terminal globular domain homologous to C1q. The metabolic and insulin-sensitizing effects of adiponectin can be at least partially explained by their direct activation of AMPK, leading to increased fatty acid oxidation and the prevention of triacylglycerol accumulation and lipotoxicity in these tissues [12,14].

Adiponectin is secreted specifically from adipocytes: Different complexes build up from a low molecular weight (LMW) trimer form, to middle molecular weight (MMW) hexamers consisting of two trimers, to high molecular weight (HMW) forms consisting of up to 18 molecules [13]. Glucose tolerance is better correlated with the levels of HMW complexes in the serum than with total adiponectin [13]. Improvement of insulin sensitivity in treated human diabetics was associated with an increase in the ratio of plasma HMW adiponectin to total adiponectin levels. A relative increase in the $\mathrm{HMW} / \mathrm{MMW}$ ratio and a reduced LMW adiponectin were also observed after weight reduction. Mutations of the adiponectin gene, leading to alterations in the formation of HMW complexes, are associated with type II diabetes and extremely low circulating levels of HMW adiponectin. These studies suggest that HMW adiponectin is a better biomarker of insulin resistance than is the commonly used measure of total adiponectin [13]. Reduced adiponectin levels associated with gene polymorphisms have been linked to metabolic syndrome, type II diabetes, and coronary artery disease.

Since administration of adiponectin to persons suffering from these diseases could potentially ameliorate their symptoms we thought of producing the protein in baculovirus as this system executes eukaryotic post-translation modifications, and in Escherichia coli, for bacteria can express a larger amount of protein. We found baculovirus adiponectin to be more active, particularly in what concerns human preadipocyte differentiation. Presumably, the protein is more active because it can form larger multimers than its $E$. coli counterpart, thus, baculovirus adiponectin is a better candidate to use in future human therapy of type II diabetes, obesity, atherosclerosis, and hypertension.

\section{Materials and methods}

Reagents

Chemicals were from Sigma-Aldrich (St. Louis, MO). Cells were obtained from ECACC and cell culture and transfection reagents were from Invitrogen (Carlsbad, CA) as was pFastBac and sf9 cells. pET 25 was purchased from Novagen (Merck Biosciences, Nottingham). Ni-NTA resin, plasmid miniprep kits, and his-tag purification systems were from Qiagen (Hilden, Germany). Enzymes for molecular biology were obtained from Promega (Madison, WI) or New England Biolabs (Ipswich, MA). The human adiponectin cDNA was purchased from Open Biosystems (Hunstville, AL). Electrophoresis equipment was from Bio-Rad Laboratories (Hercules, CA).
Primary human adipocyte culture

Primary human adipocytes from the ECACC were cultured in DMEM (Gibco) supplemented with $33 \mu \mathrm{M}$ biotin, $17 \mu \mathrm{M}$ pantothenate, $10 \mu \mathrm{g} / \mathrm{ml}$ transferrin, $2 \mathrm{mM}$ L-glutamine, $100 \mathrm{U} / \mathrm{ml}$ penicillin, and $100 \mu \mathrm{g} / \mathrm{ml}$ streptomycin. Cultures were incubated at $37{ }^{\circ} \mathrm{C}$ with $5 \%$ carbon dioxide.

\section{Bacterial expression of adiponectin}

Human adiponectin cDNA was cloned into pET25b and transformed into BL21(DE3)pLys-S cells for inducible expression. Expression cultures $(10 \mathrm{ml})$ were inoculated from an overnight starter culture and allowed to reach an $A_{600}$ of 0.6 before induction with $1 \mathrm{mM}$ IPTG for $3 \mathrm{~h}$ at $37^{\circ} \mathrm{C}$. Cells were either resuspended in SDS-PAGE sample buffer or extracted with a Qiagen non-denaturing lysis buffer. Samples were cleared by centrifugation at $16,000 \mathrm{~g}$ for $20 \mathrm{~min}$ at $4{ }^{\circ} \mathrm{C}$ and analyzed by SDS or native PAGE.

\section{Baculovirus expression of adiponectin}

Human adiponectin cDNA was cloned in pFastBac (Invitrogen) after insertion of a his-tag sequence in the vector. This construct was transfected into sf9 cells in culture, following the protocols provided by the manufacturer. Adiponectin expression was followed by SDS-PAGE of the cells and the culture supernatant.

\section{Purification of recombinant adiponectin}

Adiponectin-His was purified by affinity chromatography nickel columns using the materials, columns, and protocols provided by Qiagen.

\section{Analysis of monomeric and multimeric adiponectin}

Adiponectin multimers were separated by PAGE, according to protocols provided by Bio-Rad. Gels were stained with Coomassie blue R-250.

\section{Results}

Expression of human adiponectin in E. coli and baculovirus

Human adiponectin cDNA was purchased from Open Biosystems and subcloned into the $E$. coli expression vector pET25 (Novagen) in frame with the histidine tag. Expression in bacteria was induced with either 0.5 or $1 \mathrm{mM}$ IPTG (Fig. 1A, lanes 3 and 4). Both IPTG concentrations were found to be effective for adiponectin expression (Fig. 1A) irrespective of the induction time (results not shown). Induced cells were collected by centrifugation and human adiponectin was purified on Ni-NTA columns (Qiagen) using a native procedure (see Materials and methods). The protein was deemed pure by SDS-PAGE followed 


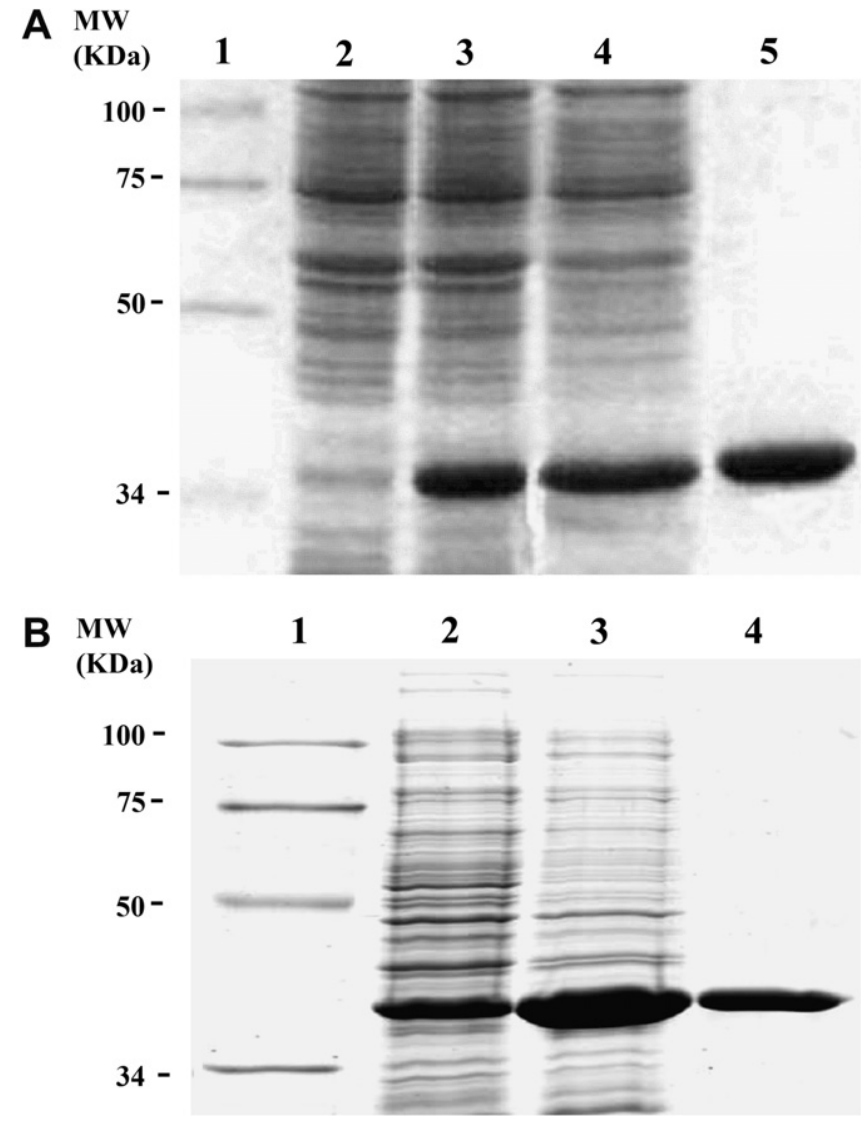

Fig. 1. Expression of adiponectin in E. coli (A) and baculovirus (B) assessed by SDS-PAGE. (A) Lane 1-Molecular weight markers; lane 2total extract from $E$. coli prior to induction; lanes 3 and 4 induction of adiponectin expression by 0.5 or $1 \mathrm{mM}$ IPTG, respectively; lane 5purified recombinant adiponectin. (B) Lane 1-Molecular weight markers; lanes 2 and 3 -expression of adiponectin in baculovirus after $36 \mathrm{~h}$ or $72 \mathrm{~h}$ Sf9 cell infection (respectively); lane 4-purified adiponectin from baculovirus. For cell extracts $10 \mu \mathrm{l}$ of sample per lane was used, whereas $1 \mu \mathrm{g}$ of purified protein was loaded. Gels were stained with Coomassie Blue-R 250 .

by Coomassie staining (Fig. 1A, lane 5). The yield was typically $10-20 \mathrm{mg}$ of protein per $250 \mathrm{~mL}$ of culture.

The same cDNA was subcloned into pFastBac (Invitrogen) following insertion of a his-tag sequence into the vector. Positive recombinant bacmids were selected and used to transfect Sf9 cells in culture following the protocols provided by Invitrogen. The virus was amplified by Sf9 cell infection and collection of the culture supernatant. Adiponectin expression was assessed by SDS-PAGE after 36 or $72 \mathrm{~h}$ of infection (Fig. 1B, lanes 2 and 3). The fusion (histag) protein was purified from $36 \mathrm{~h}$ infected Sf9 culture supernatant by affinity chromatography on Ni-NTA columns (Qiagen) and its purity assessed by SDS-PAGE (Fig. 1B, lane 4). Typical yields were of $10 \mathrm{mg}$ of protein per liter of $\mathrm{Sf} 9$ cell culture.

\section{Differentiation of pre-adipocytes}

Adiponectin has the ability to induce differentiation of pre-adipocytes into mature adipocytes as upon binding to its receptor it stimulates a transcriptional cascade resulting in a gene expression profile specific for adipocyte function. Several critical transcription factors and cross-talk between those factors have been well defined, including the CCAAT/enhancer-binding proteins (C/EBP) and peroxisome proliferators-activated receptor-gamma (PPAR- $\gamma$ ). The phosphoinositide-3-kinase (PI3K)/Akt pathway has also been shown to be critically involved in adipocyte differentiation.

In order to investigate the activity of $E$. coli and baculovirus produced adiponectin we chose to grow human preadipocytes (obtained from the ECACC) and check the ability of the protein to induce its differentiation. Differentiation was estimated by viewing the morphology of the cells (Fig. 2) which changes from a fibroblast-like figure (Fig. 2A) to round cells with lipid droplets stained by neutral red (Fig. 2B-D). Cells were then counted in a Neubauer chamber.

Adiponectin produced in baculovirus was far more active in promoting differentiation than adiponectin produced in E. coli (Figs. 2B-D and 3) as lower concentrations of the protein were able to induce full maturation of preadipocytes. To achieve the same degree of differentiation, $10 \times$ more $E$. coli protein was needed, presumably due to the post-translation modifications a baculovirus system is able to carry on (Fig. 3).

\section{Analysis of adiponectin multimers}

As adiponectin exists in vivo as homomultimers and the HMW (high molecular weight) form is more active in what concerns conferring insulin sensitivity to cells [16], we sought for the multimeric forms of both kinds of adiponectin in solution. Fig. 4 depicts a gel where adiponectin homomultimers are separated by PAGE. Remarkably, baculovirus adiponectin is able to form multimers up to 18 molecules in size (lane B), whereas E. coli adiponectin forms mainly trimers (lane $\mathrm{C}$ ). In both cases, monomeric adiponectin is the mainly species seen. We presume that

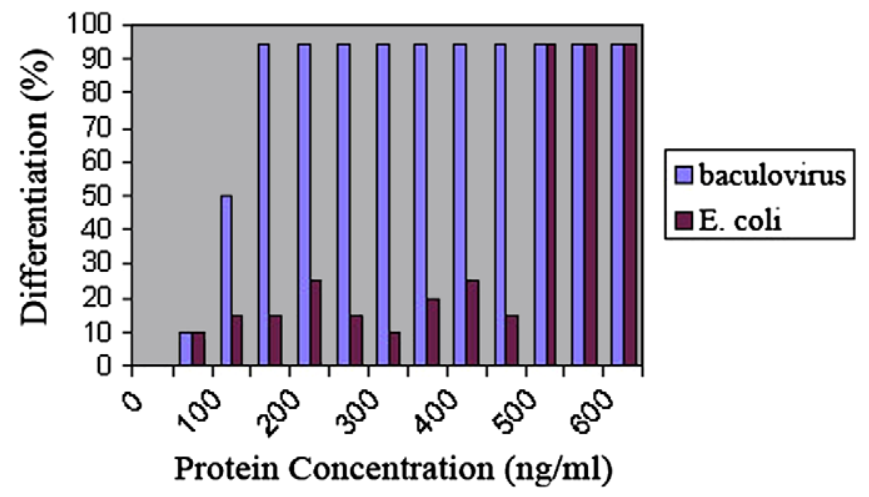

Fig. 2. Contrast phase microscopy images of human pre-adipocytes and adipocytes in culture (amplification $-400 \times$ ). (A) Pre-adipocytes, (B) Adipocytes differentiated by addition of $0.5 \mu \mathrm{g} / \mathrm{ml}$ adiponectin expressed in $E$. coli, (C and D) Adipocytes differentiated by addition of adiponectin produced in baculovirus $(\mathrm{C}, 0.1 \mu \mathrm{g} ; \mathrm{D}, 0.5 \mu \mathrm{g})$. 

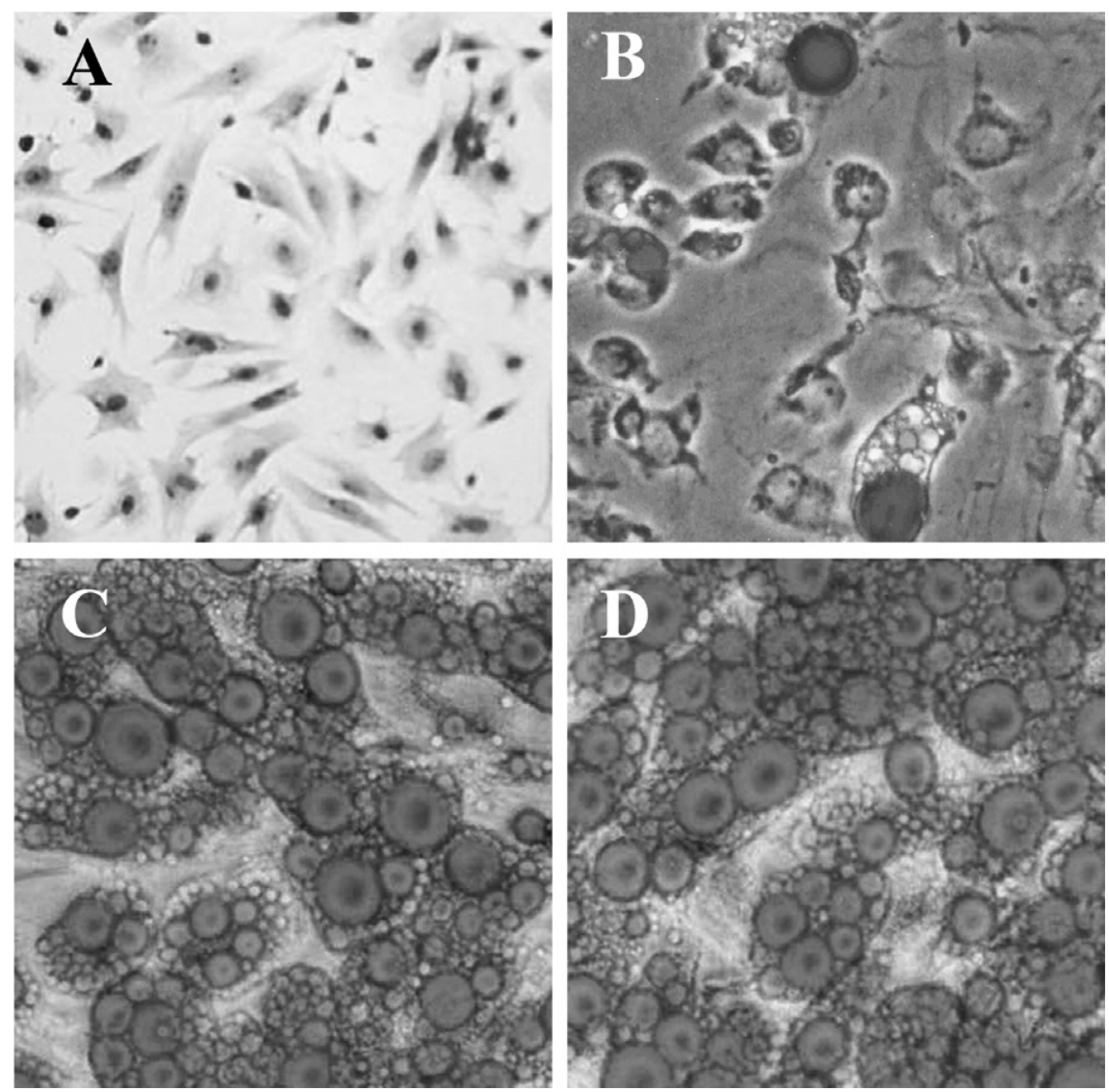

Fig. 3. Quantification of differentiated adipocytes after addition of purified adiponectin to pre-adipocytes in culture.

the ability of adiponectin to self-associate depends on posttranslation modifications not possible in E. coli [16].

\section{Discussion}

Adiponectin is expressed exclusively by differentiated adipocytes and its expression is induced during adipocyte differentiation [3]. Plasma levels of adiponectin are reduced in obese/diabetic mice and humans, in patients with cardiovascular diseases, hypertension, and metabolic syndrome but the mechanism of reduction on the plasma adiponectin level is not clearly understood [4]. Reduction of adiponectin levels is one of the causes of type II diabetes as mice deficient for adiponectin are insulin resistant and replenishment of a physiological dose of recombinant adiponectin to these mice significantly ameliorates insulin resistance [4]. Moreover, insulin resistance in lipoatrophic mice is completely reversed by the combination of physiological doses of adiponectin and leptin [4]. These data indicate that adiponectin has a direct insulin-sensitizing action and suggest that adiponectin and leptin are the two major insulin-sensitizing hormones secreted by adipose tissue. Plasma adiponectin levels are also decreased in an obese rhesus monkey model that frequently develops type II diabetes and have also been reported to be decreased in obese humans, and to correlate inversely with insulin resistance [3]. Hypoadiponectinemia is also commonly observed in a variety of states frequently associated with insulin resistance, such as cardiovascular disease and hypertension. Thus, administration of adiponectin by spray aspiration or injection can potentially help patients with these disorders.

A therapeutic target for the treatment of insulin resistance, type II diabetes, the metabolic syndrome, and cardiovascular disease may include the upregulation of plasma adiponectin levels (e.g. by the administration of recombinant adiponectin), the upregulation of adiponectin receptors, or the development of adiponectin receptors agonists.

Potentially, one could administer either adiponectin produced in E. coli or baculovirus and so we thought of producing and purifying the hormone in both systems. Adiponectin is readily expressed in both, although expression in baculovirus is demanding and time-consuming. Despite this drawback, the protein is more active as far as pre-adipocyte maturation is concerned when expressed in baculovirus, presumably due to the post-translation modifications carried out in this system. It is also possible that adiponectin expressed in E. coli is not folded correctly and cannot self-associate. Post-translation modifications of adiponectin, such as hydroxylation of multiple conserved proline and lysine residues and glycosylation of hydroxylysines, have been shown to exist in vivo and to be required for recombinant adiponectin oligomerization [16]. Indeed, we found baculovirus produced adiponectin to exist in multimeric species similar to the ones described as active. 


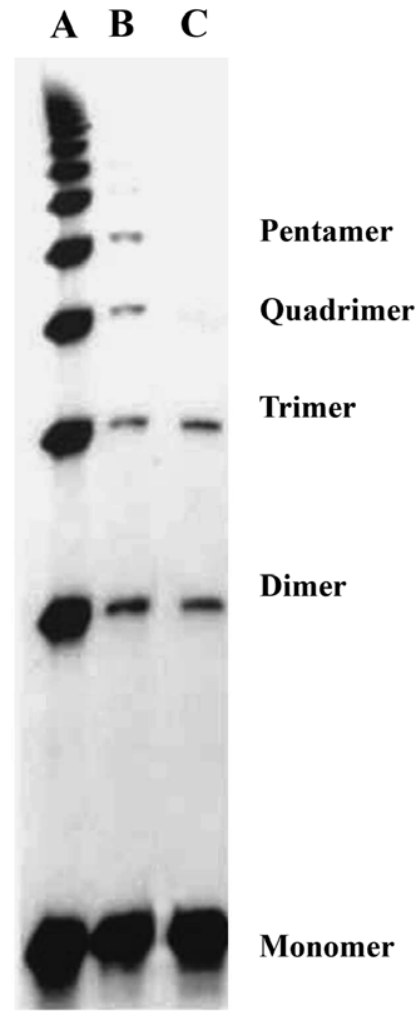

Fig. 4. Separation of adiponectin homomultimers on a PAGE gel. (Lane A) Molecular weight markers; (lane B) adiponectin from baculovirus; (lane C) adiponectin from $E$. coli. Ten micrograms of protein was used in each lane. The gel was stained with Coomassie Blue R-250.

These multimers should be required to restore adiponectin activity and so we believe that therapeutical use of largescale baculovirus produced adiponectin might be beneficial for type II diabetes and cardiovascular disease.

\section{Acknowledgments}

We are grateful to Alvaro Tavares and Claudia Florindo (Instituto Gulbenkian de Ciência) for the help with cell lines. Maria do Carmo Avides holds a Post-Doctoral Fellowship from FCT (SFRH/BPD/26913/2006).

\section{References}

[1] J.S. Flier, Obesity wars: molecular progress confronts an expanding epidemic, Cell 16 (2004) 337-350.

[2] J.M. Friedman, Obesity in the new millennium, Nature 404 (2000) 632-634.

[3] G.M. Reaven, Hypothesis: muscle insulin resistance is the ("not-so") thrifty genotype, Diabetologia 41 (1998) 482-484.

[4] N.M. Kaplan, The deadly quartet. Upper-body obesity, glucose intolerance, hypertriglyceridemia, and hypertension, Arch. Intern. Med. 149 (1989) 1514-1520.

[5] H. Masukazi, J. Paterson, H. Shinyama, N. Morton, J.J. Mullins, J.R. Seckl, J.S. Flier, A transgenic model of visceral obesity and the metabolic syndrome, Science 5549 (2001) 2166-2170.

[6] T. Kowaki, T. Yamauchi, N. Kubota, K. Hara, K. Ueki, K. Tobe, Adiponectin and adiponectin receptors in insulin resistance, diabetes, and the metabolic syndrome, J. Clin. Invest. 116 (2006) 1784-1792.

[7] E.E. Kershaw, J.S. Flier, Adipose tissue as an endocrine organ, J. Clin. Endocrinol. Metab. 89 (2004) 2548-2556.

[8] P. Cornelius, O.A. MacDougal, M. Lane, Regulation of adipocyte development, Annu. Rev. Nutr. 14 (1994) 99-129.

[9] X. Wang, Z. Hu, J. Hu, J. Du, W.E. Mitch, Regulation of adipocyte development, Annu. Rev. Nutr. 14 (1994) 99-129.

[10] A.H. Berg, T.P. Combs, X. Du, M. Braunlee, P.E. Scherer, The adipocyte-secreted protein Acrp30 enhances hepatic insulin action, Nat. Med. 7 (2001) 947-953.

[11] T.P. Combs, A.H. Berg, S. Obici, P.E. Scherer, L. Rossetti, Endogenous glucose production is inhibited by the adipose-derived protein Acrp30, J. Clin. Invest. 108 (2001) 1781-1785.

[12] T. Yamauchi et al., Adiponectin stimulates glucose utilization and fatty-acid oxidation by activating AMP-activated protein kinase, Nat. Med. 8 (2002) 1288-1295.

[13] E. Tomas, T.S. Tsao, A.K. Saha, H.E. Murrey, Cc C Zhang, S.I. Itani, H.F. Lodish, N.B. Ruderman, Enhanced muscle fat oxidation and glucose transport by ACRP30 globular domain: acetyl-CoA carboxylase inhibition and AMP-activated protein kinase activation, Proc. Natl. Acad. Sci. USA 99 (2002) 16309-16313.

[14] T.P. Combs, A.H. Berg, S. Obici, P.E. Scherer, L. Rossetti, Endogenous glucose production is inhibited by the adipose-derived protein Acrp30, J. Clin. Invest. 108 (2001) 1875-1881.

[15] R.S. Ahima, Adipose tissue as an endocrine organ, Obesity (Silver Spring) 14 (2006) 242S-249S

[16] A.A. Richards, T. Stephens, H.K. Charlton, A. Jones, G.A Macdonald, I.B. Prins, J.P. Whitehead, Adiponectin multimerization is dependent on conserved lysines in the collagenous domain: evidence for regulation of multimerization by alterations in post-translation modifications, Mol. Endocrinol. 20 (2006) 1673-1687. 